\title{
Effects of nanoencapsulation and PEGylation on biodistribution of indocyanine green in healthy mice: quantitative fluorescence imaging and analysis of organs
}

\author{
This article was published in the following Dove Press journal: \\ International Journal of Nanomedicine \\ 19 April 2013 \\ Number of times this article has been viewed
}

\author{
Baharak Bahmani' \\ Christian Y Lytle ${ }^{2}$ \\ Ameae MWalker ${ }^{2}$ \\ Sharad Gupta' \\ Valentine IVullev' \\ Bahman Anvari' \\ 'Department of Bioengineering, \\ ${ }^{2}$ Division of Biomedical Sciences, \\ University of California, Riverside, \\ CA, USA
}

Correspondence: Bahman Anvari University of California, Riverside, Department of Bioengineering, Riverside, California 9252I, USA

Tel + I 95I 8275726

Fax + I 95I 8276416

Email anvarib@ucr.edu

\begin{abstract}
Near-infrared nanoconstructs present a potentially effective platform for site-specific and deep tissue optical imaging and phototherapy. We have engineered a polymeric nanocapsule composed of polyallylamine hydrochloride (PAH) chains cross-linked with sodium phosphate and doped with indocyanine green (ICG) toward such endeavors. The ICG-doped nanocapsules were coated covalently with polyethylene glycol (5000 daltons) through reductive amination. We administrated the constructs by tail vein injection to healthy mice. To characterize the biodistribution of the constructs, we performed in vivo quantitative fluorescence imaging and subsequently analyzed the various extracted organs. Our results suggest that encapsulation of ICG in these PEGylated constructs is an effective approach to prolong the circulation time of ICG and delay its hepatic accumulation. Increased bioavailability of ICG, due to encapsulation, offers the potential of extending the clinical applications of ICG, which are currently limited due to rapid elimination of ICG from the vasculature. Our results also indicate that PAH and ICG-doped nanocapsules (ICG-NCs) are not cytotoxic at the levels used in this study.
\end{abstract}

Keywords: cancer, fluorescent imaging, nanoprobes, near infrared, pharmacokinetics, phototherapy, vascular imaging

\section{Introduction}

Nanoconstructs containing near-infrared (NIR) exogenous chromophores are currently being investigated as enabling platforms for site-specific optical imaging. ${ }^{1-3}$ Use of NIR wavelengths is particularly advantageous, in that it allows for relatively deep optical penetration, in the order of a few centimeters in biological tissues, due to reduced light absorption by water and proteins. ${ }^{4}$ Additionally, tissue autofluorescence is negligible over the NIR spectral band, allowing for increased contrast when using exogenous NIR fluorophores. ${ }^{5,6}$

We have engineered polymeric nanocapsules composed of polyallylamine hydrochloride (PAH) chains cross-linked with sodium phosphate, and doped with indocyanine green (ICG), the only US Food and Drug Administration-approved NIR chromophore for specific imaging applications. ${ }^{7-11}$ For example, in ophthalmology, ICG is used to image the nature of choroidal circulation and its abnormalities including suspected polypoidal choroidal neovascularization, choroidal hemangioma, chronic central serous chorioretinopathy, and certain forms of neovascularization in age-related macular degeneration. ${ }^{12-14}$ Other clinical applications of ICG are in assessment of cardiovascular and liver functions. ${ }^{15,16}$ ICG has also been investigated in patients with breast, gastric, 
and skin cancer undergoing sentinel lymph node mapping, ${ }^{17-23}$ and evaluation of lymphedema. ${ }^{24,25}$

Despite its proven efficacy in these applications, the utility of ICG for broader clinical usage has been limited due to its nonspecific binding to albumin and high-density lipoproteins in plasma. Such binding results in rapid clearance of ICG from blood (with plasma half-life in the order of 2-4 minutes). ${ }^{26} \mathrm{We}^{7-11}$ and others ${ }^{27-30}$ are pursuing encapsulation of ICG as a potential approach to increase its circulation time with the intention of broadening the medical applications of this clinically proven optical material.

A common phenomenon associated with the use of exogenous nanoconstructs is that, once administered into the vasculature, blood plasma proteins can bind to the surface of these materials. Macrophages of the reticuloendothelial system recognize the nanoparticles by the presence of these surface-adsorbed proteins, and clear them from the vasculature through phagocytosis. ${ }^{31}$ The circulation time of nanoparticles within the vasculature can be improved by minimizing nonspecific protein binding and phagocytic recognition. Development of bio-inert (non-fouling) surfaces that resist protein adsorption is a common strategy to prolong the vascular circulation time of nanoparticles. ${ }^{32}$

In the study reported here, we modified the surface of ICG-doped nanocapsules (ICG-NCs) with polyethylene glycol (PEG) to increase the circulation time of the constructs. In a previous study, we demonstrated that PEGylating ICG-NCs reduced the uptake of the constructs by primary human hepatocytes in vitro. ${ }^{33}$ We have also investigated the effect of low $(5 \mathrm{kDa})$ and high $(30 \mathrm{kDa})$ molecular weights (MW) of PEG coating, and determined that coating the surface of ICG-NCs with $5 \mathrm{kDa}$ PEG reduces the interaction between the particles and spleen macrophages, as determined by flow cytometry. ${ }^{10}$ Motivated by these previous in vitro studies, in the study reported here, we extended our investigations to in vivo studies using healthy mice. Specifically, the objectives of this study were to evaluate the effectiveness of encapsulation and coating the surface of ICG-NCs with $5 \mathrm{kDa}$ PEG in extending the circulation time of ICG and to investigate the utility of these ICG-NCs for in vivo quantitative fluorescence imaging.

\section{Methods}

\section{Synthesis and PEGylation of ICG-NCs}

ICG-NCs were synthesized by ionic cross-linking PAH chains to sodium phosphate and then loading the nanocapsules with ICG using diffusion mediation, as reported previously. ${ }^{7-11,34,35}$ The amounts of PAH and sodium phosphate and the aging time controlled the size of the ICG-NCs. ${ }^{9}$ Briefly, we fabricated the ICG-NCs by mixing $20 \mu \mathrm{L}$ of PAH stock solution $\left(2 \mathrm{mg} / \mathrm{mL}, 4^{\circ} \mathrm{C}\right)$ and $10 \mu \mathrm{L}$ of disodium hydrogen phosphate heptahydrate solution $\left(0.01 \mathrm{M}, 4^{\circ} \mathrm{C}\right)$. The nanoparticle suspension was then diluted by the addition of $1.2 \mathrm{~mL}$ pre-cooled deionized water $\left(4^{\circ} \mathrm{C}\right)$, and this was immediately followed by the addition of $240 \mu \mathrm{L}$ of ICG aqueous solution $\left(0.65 \mathrm{mM}, 4^{\circ} \mathrm{C}\right)$. The ICG-NC suspension was aged for 15 minutes at $4^{\circ} \mathrm{C}$, and then washed through differential centrifugation. The suspension was centrifuged at $2300 \mathrm{~g}$ (5000 rpm) for 5 minutes followed by another centrifugation at $845 \mathrm{~g}(3000 \mathrm{rpm})$ for 30 minutes to separate out the large ICG-NCs. Then ICG-NCx were washed twice and collected using centrifugation after each wash ( $845 g$ for 2 hours).

The ICG-NCs were subsequently coated with aldehydeterminated polyethylene glycol (PEG-ALD, MW = 5000 Da) using reductive amination, as previously described. ${ }^{10}$ The PEG-ALD was added to the ICG-NC suspension to conjugate one PEG chain $/ \mathrm{nm}^{2}$ of the nanoparticle surface. We used approximately two equivalents of sodium dithionite per mole PEG-ALD as a reducing agent. This suspension was then aged for 2 hours at $4^{\circ} \mathrm{C}$. This process resulted in the formation of a covalent bond between the amine groups on the surface of the ICG-NCs and the aldehyde group of the PEG-ALD. The PEG-coated ICG-NCs were washed twice and collected by centrifugation after each wash ( $845 g$ for 2 hours). The uncoated and PEG-coated ICG-NCs were resuspended in phosphate-buffered saline and stored at $4{ }^{\circ} \mathrm{C}$ in the dark.

\section{Characterization of ICG-NCs}

The morphology of the ICG-NCs was determined using scanning electron microscopy (XL-30 FEG, Philips, Amsterdam, Netherlands). Hydrodynamic diameters of the uncoated and PEGylated ICG-NCs were measured by dynamic light scattering (Zetasizer Nanoseries, NanoZS90, Malvern Instruments, Malvern, UK). Absorption spectra of ICG-NCs were obtained using a Cary $50 \mathrm{UV}-$ Vis spectrophotometer (Agilent Technologies, Santa Clara, CA, USA) with a $1 \mathrm{~cm}$ path length. Fluorescence spectra of the ICG-NCs were obtained with a Fluorolog-3 spectrofluorometer (Edison, NJ, USA) in response to a $680 \mathrm{~nm}$ excitation wavelength, a wavelength at which both non-PEGylated and PEGylated constructs have nearly the same absorbance value. For comparison, we obtained the absorption spectrum of $9 \mu \mathrm{M}$ ICG dissolved in water, and its corresponding fluorescence spectrum in response to $680 \mathrm{~nm}$ excitation. 


\section{Cytotoxicity assessment}

We incubated human dermal microvascular endothelial cells (PCS-110 cell line) purchased from the American Type Culture Collection (Manassas, VA, USA) with media containing uncoated ICG-NCs $(4.6 \mu \mathrm{g}$ PAH/mL), PEG-coated ICG-NCs $(4.6 \mu \mathrm{g} \mathrm{PAH} / \mathrm{mL}$ ), or PAH (at 6 and $12 \mu \mathrm{g} / \mathrm{mL}$ ) for 24 hours at $37^{\circ} \mathrm{C}$ and $5 \% \mathrm{CO}_{2}$. Cells without exposure to any agent, and those incubated with phenol $(100 \mu \mathrm{L})$ were used as negative and positive controls, respectively. After 24 hours, cells were washed twice with phosphate-buffered saline and stained with propidium iodide to identify the dead cells. We used flow cytometry to determine the percentage of dead cells present.

\section{Animal preparation and administration of imaging agents}

Female Swiss Webster mice, 25-30 g, 10-12 weeks old, were procured from Charles River Laboratories (Wilmington, MA, USA) and utilized in this study under a protocol (A-20080039) approved by the University of California Riverside Institutional Animal Care and Use Committee. Animals were anesthetized by inhalation of $2 \%$ isoflurane in oxygen. The ventral side of each mouse was shaved 1 hour prior to administration of ICG-NCs. Following this, PEG-coated ICG-NCs, uncoated ICG-NCs, or freely dissolved ICG were administered intravenously via tail vein injection while the animal was anesthetized. The absorbance values of all samples at $800 \mathrm{~nm}$ were the same and equivalent to the value associated with $12 \mu \mathrm{M}$ of free ICG. The administered level of ICG in our experiments, $75 \mu \mathrm{g} \mathrm{ICG} / \mathrm{kg}$ weight of mouse, was well below the lethal dosage in $50 \%$ of animals of $62 \mathrm{mg} / \mathrm{kg}$ in mice. ${ }^{36}$ The injection volume for all samples was $150 \mu \mathrm{L}$.

\section{Whole-body fluorescence imaging}

Whole-body images of the mice were captured using a luminescence dark box. Each animal was placed in a supine position on a heating pad to maintain body temperature. A constant flow of $2 \%$ isoflurane in oxygen was delivered using a plastic gas manifold during the imaging to immobilize the mice. Images were captured at various times and up to 60 minutes post-injection of samples. Two light emitting diodes (LEDs) equipped with an excitation filter $(700 \pm 30 \mathrm{~nm})$ were used for illumination. Fluorescent emission was captured using a charge-coupled device camera (Pixis 1024B, Roper Scientific, Trenton, NJ, USA) equipped with a long-pass filter transmitting wavelengths greater than $810 \mathrm{~nm}$. To prevent pixel saturation, the camera exposure time was set to 0.5 second for animals injected with free ICG and 1.0 second for animals injected with other agents.

\section{Quantitative image analysis}

The acquired fluorescent images were analyzed using Image J software. ${ }^{37}$ The abdominal area of the mouse, corresponding to the liver and small intestine, was selected as the region of interest (ROI). The mean intensity $(I)$ values of each fluorescent image acquired from the ROI at different post-injection times were calculated as:

$$
\bar{I}=\frac{\sum_{j=1}^{m} I_{j}}{m},
$$

where $m$ is the total number of pixels in the ROI, and $I_{j}$ is the pixel intensity at the jth pixel. Subsequently, we computed the image contrast $(C)$ associated with the ROI as:

$$
C=\frac{\bar{I}_{T}-\bar{I}_{B}}{\bar{I}_{B}}
$$

where $\bar{I}_{T}$ and $\bar{I}_{B}$ represent the mean intensity of the ROI (abdominal area) and background, respectively. ${ }^{8,38}$

\section{Biodistribution characterization}

Mice were euthanized by inhalation of compressed $\mathrm{CO}_{2}$ gas at various times $(15,30$, and 60 minutes) following injection with PEG-coated ICG-NCs, uncoated ICG-NCs, or freely dissolved ICG. Five mice were studied for each of the imaging agents and each time point, giving a total of 45 animals. After sacrificing each mouse, $500 \mu \mathrm{L}$ of blood was collected from the heart by cardiac puncture. The blood sample was mixed with $5 \mathrm{~mL}$ of sodium dodecyl sulfate (SDS, $5 \% \mathrm{w} / \mathrm{v}$ in water) solution to lyse the blood cells, and release the ICG molecules or nanocapsules taken up by the cells. Various organs including the heart, lungs, liver, kidneys, spleen, stomach, and intestine were harvested at each time point and for each imaging agent. Organs were ground using disposable polystyrene tissue grinders (Fisherbrand, Fisher Scientific International, Hampton, NH, USA). Smaller organs, such as the heart, spleen, lungs, and kidneys, were incubated in $5 \mathrm{~mL}$ of SDS solution for 1 hour to lyse the cells. The liver, intestine, and stomach were incubated in $10 \mathrm{~mL}$ of SDS solution ( $5 \% \mathrm{w} / \mathrm{v}$ in water) for 1 hour.

Lysed organs and blood samples were centrifuged in SDS solution at $12,000 \mathrm{~g}(9000 \mathrm{rpm})$ for 30 minutes at $4^{\circ} \mathrm{C}$. Subsequently, the supernatants of the blood sample and homogenized organs were collected, and the fluorescence 
emission spectrum of each sample was recorded using the fluorometer in response to an excitation wavelength of $680 \mathrm{~nm}$. The ICG content within each organ was calculated by comparing the integrated fluorescent signal (over the bandwidth of 700-900 $\mathrm{nm}$ ) with a calibration curve that related the fluorescence emission over the same bandwidth to various concentrations of ICG in SDS solution.

\section{Results}

\section{Characterization of ICG-NCs}

Scanning electron microscopy revealed the spherical morphology of the ICG-NCs (Figure 1A). Using dynamic light scattering, the peak diameter of the uncoated ICG-NCs - based on the amounts of the reagents utilized, time, and other experimental protocols, as indicated in the Methods section - was 77 nm (Figure 1B). PEGylation increased the peak diameter by nearly $10 \mathrm{~nm}$ and resulted in a right shift in the population distribution. Both peak values were associated with $23 \%$ of the nanoparticles in the population distribution.

In comparison to the absorption spectrum of free ICG, the spectra of ICG-NCs were broadened and did not show distinct peaks corresponding to the monomeric and dimeric forms of ICG at 780 and $720 \mathrm{~nm}$, respectively. This spectral broadening is consistent with our previous report. ${ }^{9}$ Uncoated and PEG-coated ICG-NCs demonstrated similar absorption and fluorescent emission spectra (Figure 2), suggesting that PEGylation did not profoundly change these optical properties of the constructs. Absorbance values were slightly higher for the PEGylated particles in the $700-860 \mathrm{~nm}$ range (Figure 2A). In response to $680 \mathrm{~nm}$ excitation, the fluorescence spectra for both uncoated and PEG-coated ICG-NCs illustrated peaks at nearly 720 and $790 \mathrm{~nm}$, corresponding to emission from the dimeric and monomeric forms of ICG, respectively, with
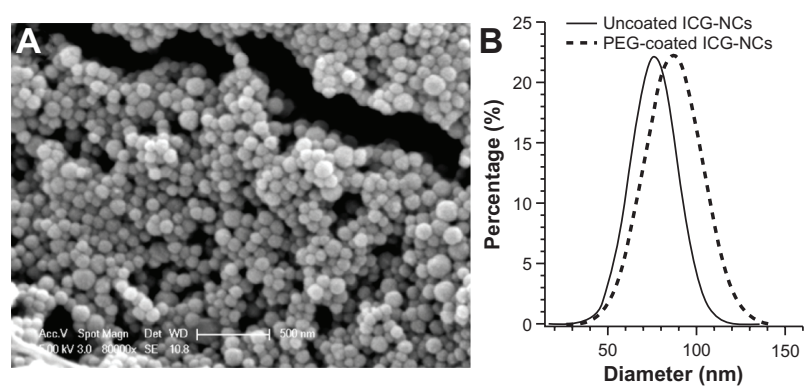

Figure I (A) Scanning electron microscope image of uncoated indocyanine greendoped nanocapsules (ICG-NCs), and (B) Gaussian fits to the diameter distribution profiles of uncoated and polyethylene glycol (PEG)-ylated ICG-NCs in water at $4^{\circ} \mathrm{C}$ as measured by dynamic light scattering. slightly higher values for the PEGylated constructs in the 720-730 nm and 750-805 nm emission bands.

We have previously reported on the improved optical and thermal stability of ICG when encapsulated in these polymeric nanocapsules. ${ }^{7}$ For example, exposure of ICG-NC suspension and free ICG to broadband light for up to 2 hours resulted in the increased degradation rate of the monomer form of free ICG from $0.0142 \mathrm{~min}^{-1}$ to $0.0042 \mathrm{~min}^{-1}$. In response to the incubation of ICG-NCs and free ICG at $40^{\circ} \mathrm{C}$ for 48 hours, the absorbance of the monomer form of free ICG decreased by $\approx 40 \%$. In contrast, the absorbance of the monomer form of ICG-NCs was reduced by only $10 \%$. $^{7}$

We have also reported the release kinetics of ICG from these polymeric nanoparticles. ${ }^{35}$ We incubated ICG-NCs in Dulbecco's modified Eagle medium supplemented with 10\% fetal bovine serum for up to 24 hours at $37^{\circ} \mathrm{C}$ in a water bath (without light exposure) to simulate a physiologically relevant environment. At various time points, the capsules were centrifuged, and the supernatants were collected and optically analyzed for ICG release. Only 5\% of ICG was released from the nanocapsules after 1 hour under these physiologically relevant conditions. At 8 hours post-incubation, the released level approached a near steady value of approximately $12 \%{ }^{35}$

In this study, we measured zeta potential values of $40 \mathrm{mV}$ and $12 \mathrm{mV}$ for non-coated ICG-NCs and PEGylated ICGNCs respectively. We have used Fourier transform infrared spectroscopy previously to confirm the covalent attachment of PEG to the surface of the ICG-NCs using the reductive amination process. ${ }^{10}$

\section{Cytotoxicity of ICG-NCs}

In Figure 3, preliminary cell viability studies using propidium iodide assay are presented. Almost $100 \%$ of the cells incubated with uncoated ICG-NCs, PEG-5k-coated ICG-NCs, and $6 \mu \mathrm{g} / \mathrm{mL}$ PAH remained viable. Cell viability was reduced to $85 \%$ after incubation with PAH at $12 \mu \mathrm{g} / \mathrm{mL}$ for 24 hours, while almost the entire cell population died when incubated with phenol. These results are very promising, since they suggest that PAH will not compromise cell viability when utilized at a concentration $<12 \mu \mathrm{g} / \mathrm{mL}$. Therefore, for these in vivo studies, we administered ICG-NCs containing PAH at concentrations $\leq 4.6 \mu \mathrm{g} / \mathrm{mL}$.

\section{Quantitative fluorescence imaging and biodistribution of constructs in mice}

The maximum fluorescence signal associated with the injection of free ICG was detectable from the liver 3 minutes post-injection (Figure 4A), indicating the rapid clearance 

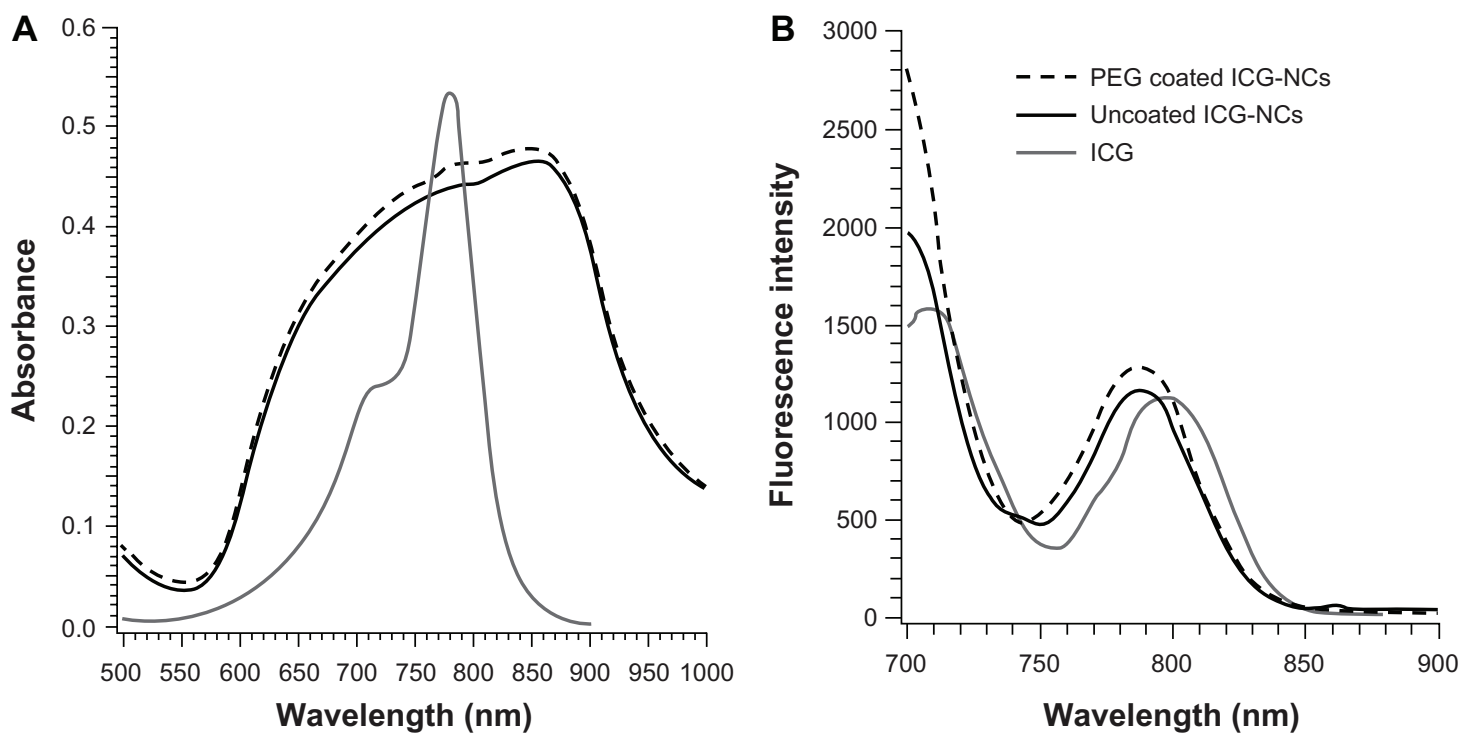

Figure 2 (A) Absorbance and (B) fluorescence spectra of indocyanine green (ICG) $(9 \mu \mathrm{M})$, uncoated and polyethylene glycol (PEG)-coated indocyanine green-doped nanocapsules (ICG-NCs) $\left(\lambda_{\mathrm{ex}}=680 \mathrm{~nm}\right)$.

of ICG from the systemic circulation, consistent with previous studies. ${ }^{39-41}$ Fluorescence emission levels from the liver remained near maximum for at least 60 minutes postinjection of free ICG (Figure 4B-E). In the case of uncoated and PEG-coated ICG-NCs, maximum fluorescence signals from the liver were detectable at 10 minutes (Figure 4G) and 60 minutes (Figure 40) post-injection, respectively.

Figure 5 shows the mean \pm standard deviation values of the image contrasts $(C)$ associated with the abdominal areas

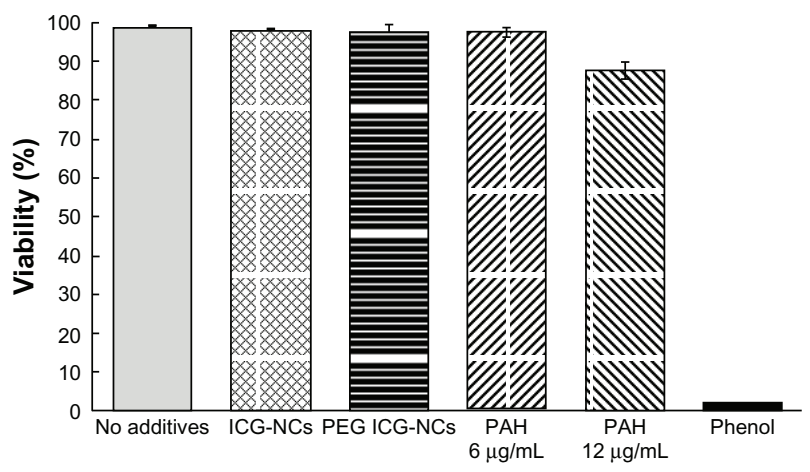

Figure 3 Viability of human dermal vascular endothelial cells after 24 hours' incubation in media containing one of the following additives: uncoated indocyanine greendoped nanocapsules (ICG-NCs; $4.6 \mu \mathrm{g} / \mathrm{mL}$ ), indocyanine green-doped polyethylene glycol-coated nanocapsules (PEG ICG-NCs, $4.5 \mu \mathrm{g} / \mathrm{mL}$ ), and polyallylamine hydrochloride (PAH) at two different concentrations.

Notes: Cells incubated in media with no additive constituted the negative control, while cells incubated in media with phenol constituted the positive control. Each bar represents the average of three experiments. Error bars represent one standard deviation. Using statistics to analyze the results for ICG-NCs and PAH $(6 \mu \mathrm{g} / \mathrm{mL})$ yielded $P$ values $>0.1$ when compared with the negative control and $P$ values $<10^{-4}$ when compared with the positive control. Therefore, these three tests indicated lack of adverse effects on cell viability. The same analysis for PAH at $12 \mu \mathrm{g} / \mathrm{mL}$ (2.6 times greater than the concentration administered in in vivo studies) yielded $P$ values $<10^{-3}$ when compared with the negative control and $P$ values $<10^{-4}$ when compared with the positive control. as the ROI at post-injection times between 3 and 60 minutes. The mean value of $C$ was about eight-fold higher for mice injected with free ICG as compared with that for mice injected with PEG-coated ICG-NCs at 3 minutes post-injection.

The dynamics of the image contrast were fitted with single exponential profiles (Figure 5), and $\tau$ was defined as the time over which the mean value of $C$ increased by a factor of $e$. The mean value of $C$ for the ROI increased rapidly up to approximately 8 minutes post-injection for free ICG ( $\tau=1 \pm 0.06$ minutes). The rapid increase of $C$ demonstrates the quick accumulation of free ICG in the liver within a short time post-injection. ICG is secreted into the bile following uptake by the liver. ${ }^{42}$ Consequently, bile that contains ICG is stored in the gallbladder and discharged to the duodenum through the bile duct. Ten minutes post-injection, the mean $C$ value for the ROI associated with free ICG (Figure 5) gradually decreased over a time interval greater than 60 minutes, suggesting either a reduced rate of accumulation in the liver or secretion of ICG into the bile, or both.

Upon injection of uncoated ICG-NCs, a different dynamic was observed; a gradual increase in the mean $C$ value for the ROI up to 60 minutes post-injection with $\tau=18.98 \pm 1.61$ minutes. The longer $\tau$ of uncoated ICGNCs compared with that associated with free ICG implies a lower clearance rate of uncoated ICG-NCs from the systemic circulation and accumulation in the liver. The mean $C$ value for the ROI associated with PEG-coated ICG-NCs gradually increased until 60 minutes post-injection, with a $\tau=34.2 \pm 1.39$ minutes (Figure 5). The mean $C$ for mice injected with PEG-coated ICG-NCs remained statistically 


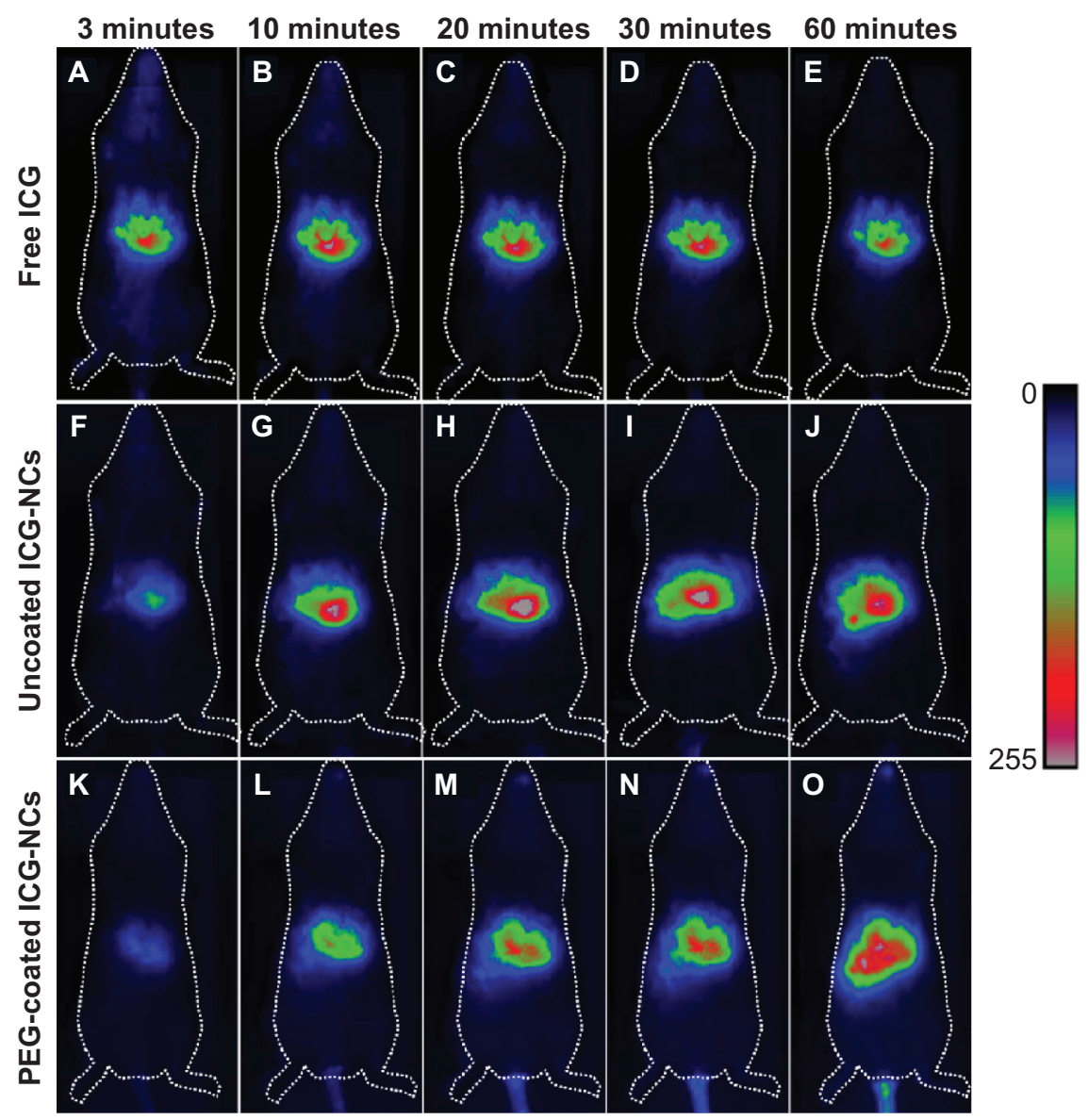

Figure 4 Whole-body fluorescent images of three different mice injected with freely dissolved indocyanine green (ICG) in phosphate-buffered saline (A-E), uncoated indocyanine green-doped nanocapsules (ICG-NCs) (F-J), and polyethylene glycol (PEG)-coated ICG-NCs (K-O) at various times post-injection.

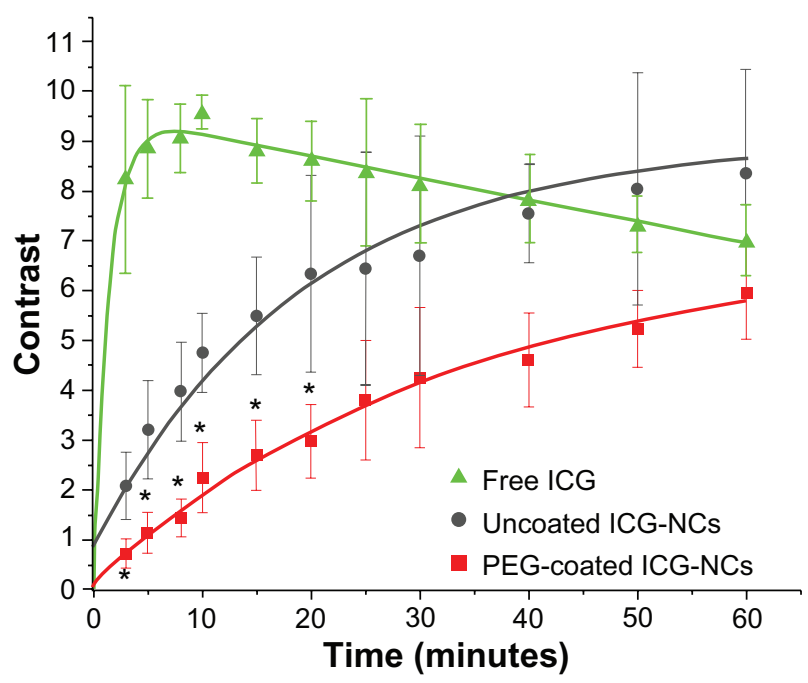

Figure 5 Computed image contrast of the abdominal area of mice injected with freely dissolved indocyanine green (ICG) in phosphate-buffered saline, uncoated indocyanine green-doped nanocapsules (ICG-NCs) and polyethylene glycol (PEG)coated ICG-NCs at various times post-injection.

Notes: The exponential fit to each dataset is presented in green (free ICG, $\mathrm{R}^{2}=0.95$ ), dark grey (uncoated ICG-NCs, $\mathrm{R}^{2}=0.96$ ) and red (PEG-coated ICG$\left.N C s, R^{2}=0.99\right)$. Error bars represent the standard deviation. Asterisks denote statistically significant differences $(P<0.05)$ between the PEG-coated ICG-NCs, uncoated ICG-NCs, and free ICG contrast. significantly lower than the corresponding values for mice injected with uncoated ICG-NCs and free ICG for up to 20 and 50 minutes post-injection, respectively. The lower mean values of $C$ and longer $\tau$ associated with PEG-coated ICGNCs imply reduced accumulation of PEGylated ICG-NCs within the liver - an indication of the prolonged circulation time of these nanoparticles.

Figure 6 shows illustrative fluorescent images of various organs obtained from six mice euthanized at 30 and 60 minutes post-injection. For mice injected with free ICG, only the liver and intestine could be fluorescently visualized, confirming that free ICG accumulated in these two organs (Figure 6A and B). For mice injected with uncoated ICG-NCs, fluorescence emission from the liver, intestine, stomach, spleen, and kidneys was detectable at 30 minutes post-injection. At 60 minutes post-injection of uncoated ICG-NCs, ICG could only be detected fluorescently in the liver, intestine, and stomach (Figure 6C and D). However, for mice injected with PEG-coated ICG-NCs, all organs except heart were distinguishable in the fluorescent images 


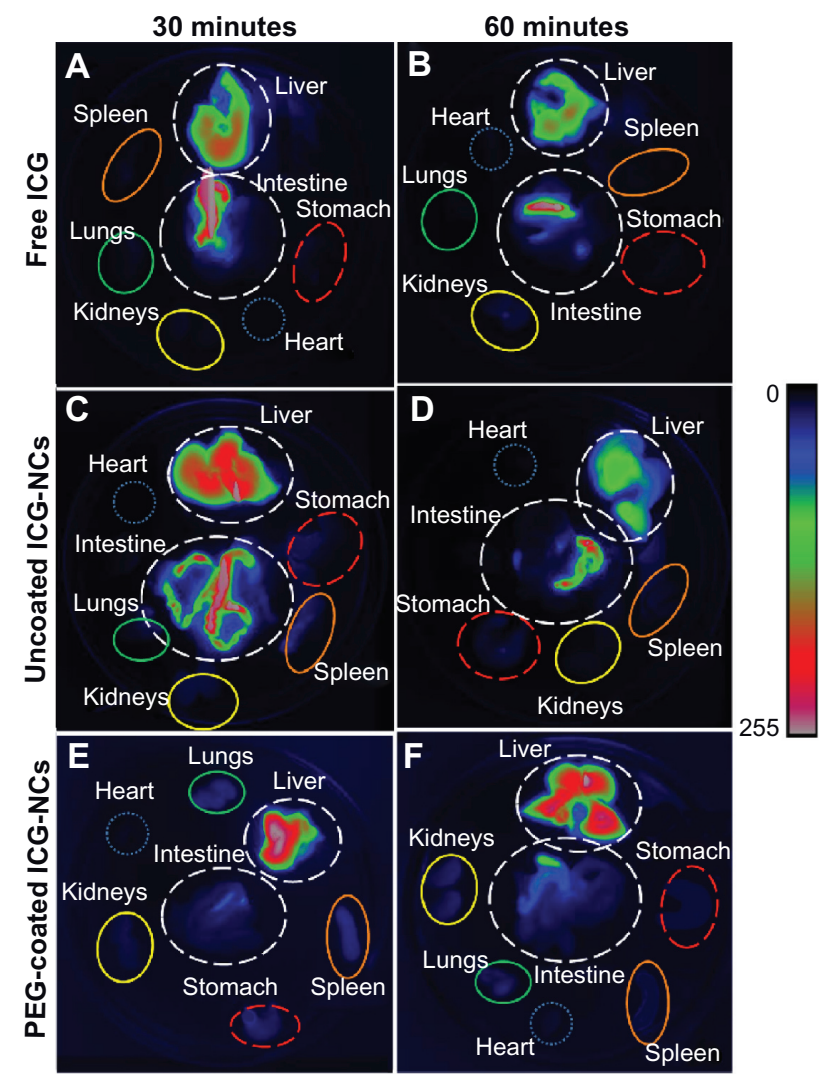

Figure 6 Fluorescent images of harvested organs of six mice at 30 and 60 minutes post tail vein administration of free indocyanine green (ICG) (A and $\mathbf{B}$ ), uncoated indocyanine green-doped nanocapsules (ICG-NCs) (C and D) and polyethylene glycol (PEG)-coated ICG-NCs (E and F).

at 30 and 60 minutes post-injection (Figure $6 \mathrm{E}$ and $\mathrm{F}$ ). The fluorescence emission detected from the highly vascularized organs including spleen, lungs, and kidneys at up to 60 minutes post-injection of PEG-coated ICG-NCs may be due to the circulating PEG-coated ICG-NCs within the vasculature or tissues of these organs.

\section{Quantification of ICG content in blood samples and harvested organs}

The relative ICG content of blood and various organs was determined at 15,30 , and 60 minutes post-injection (Figure 7). Nearly $57 \%$ of the administered PEG-coated ICG-NCs remained within the vasculature at 15 minutes post-injection (Figure 7A). This fraction was approximately three-fold higher than that for uncoated ICG-NCs and free ICG. The remainder of the PEG-coated ICG-NCs was mostly recovered from the liver (12\%), lungs (6.5\%), kidneys (5.7\%), spleen (4\%), heart (3\%), and intestine (1.7\%).

The majority of administered free ICG was recovered from blood (21.5\%), intestine (21\%), and liver (14\%) at 15 minutes post-injection. Based on the high level of ICG

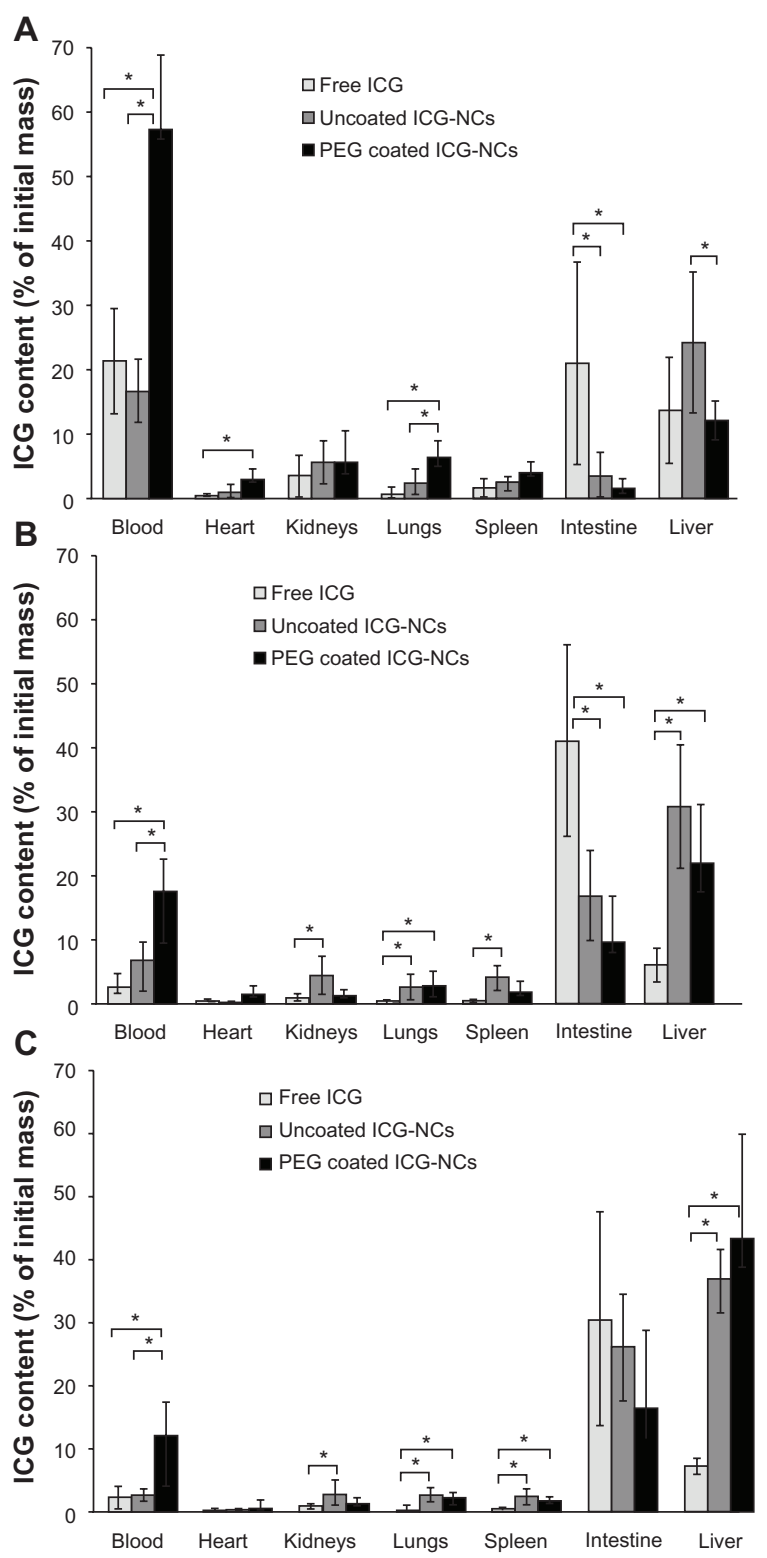

Figure 7 Estimated percentage of indocyanine green (ICG) recovered from blood, heart, kidneys, lungs, spleen, intestine, and liver of the mice injected with freely dissolved ICG in phosphate-buffered saline, uncoated indocyanine green-doped nanocapsules (ICG-NCs), and polyethylene glycol (PEG)-coated ICG-NCs at (A) I5, (B) 30, and (C) 60 minutes post-injection.

Notes: Each experiment was repeated five times. Error bars represent standard deviation. Single asterisks denote statistically significant differences of $P<0.05$ between the shown pairs.

recovered from the intestines, we suggest that the passage of free ICG from the liver to the small intestine occurred as early as 15 minutes post-injection (Figure 7A).

The ICG content in blood dropped for all three agents at longer post-injection times; nevertheless, the level of PEGcoated ICG-NCs in the blood remained significantly the highest at 15, 30, and 60 minutes post-injection as compared with uncoated ICG-NCs and free ICG. The ICG content within the blood at 30 minutes post-injection of PEG-coated 
ICG-NCs (Figure 7B) was nearly equal to the ICG content in blood at 15 minutes post-injection of free ICG or uncoated ICG-NCs (Figure 7A).

At 30 minutes post-injection, the amount of ICG in the blood and liver dropped dramatically to $7 \%$ and $6 \%$, respectively (Figure 7B); conversely, an increase in ICG content in the intestine to $41 \%$ was observed. This rise is indicative of ICG transport to the duodenum. In the case of uncoated ICG-NCs, the amount of ICG recovered from the blood, heart, and kidneys dropped to $6.8 \%, 0.3 \%$, and $4.4 \%$, respectively. However, the ICG content remained the same in the lungs and spleen. A significant increase in ICG concentration was observed in the intestine and liver at longer times post-injection of uncoated ICG-NCs (Figure 7B and C).

At 30 and 60 minutes post-injection of PEG-coated ICG$\mathrm{NCs}$, the amount of ICG dropped significantly in blood and all organs except the liver and intestine (Figure 7B and C). The dramatic increase in liver ICG content from nearly $12 \%$ at 15 minutes to $22 \%$ at 30 minutes, and $42 \%$ at 60 minutes post-injection is consistent with the whole-body fluorescent images (Figure 4) and the associated ROI image contrast (Figure 5), illustrating the delayed uptake of PEG-coated ICG-NCs by the liver. The amount of ICG recovered from the lungs, kidneys, spleen, and heart decreased at 30 and 60 minutes post-injection of PEG-coated ICG-NCs.

The spleen is one of the organs of the immune system responsible for filtering foreign body materials and dead or damaged erythrocytes from the blood. ${ }^{43}$ We noted that about $4 \%$ of the PEG-coated ICG-NCs was recovered from spleen at 15 minutes post-injection. However, the level of recovered ICG was statistically significantly reduced to $2 \%$ at 60 minutes post-injection. Therefore, we associate the high level of PEGylated ICG-NCs recovered from the spleen at 15 minutes post-injection with the nanoparticles circulating within the vasculature of spleen. In the open blood circulation system of the spleen, the capillaries discharge openly into the pulp cord before reaching the sinusoids. The pulp cords contain reticular cells, macrophages, plasma cells and lymphocytes. ${ }^{44}$ The PEGylated ICG-NCs in the blood have to pass through the pulp cord before entering the venous sinusoids. We attribute the initial rise in fluorescent signal detected from the spleen and the concentration of recovered nanocapsules from this organ to the portion of the PEGcoated ICG-NCs passing through the pulp cord and not to the uptake by splenic macrophages.

Lungs, as the organs receiving the entire cardiac output, are prone to the accumulation of nanocapsules. ${ }^{45}$ Our results demonstrate that a statistically significant level of ICG was recovered from the lungs $(6.5 \%)$ at 15 minutes post-injection of PEG-coated ICG-NCs compared with uncoated ICGNCs and free ICG (Figure 7A). The drop in ICG content in the lungs to $2.5 \%$ after 1 hour following administration of PEG-coated ICG-NCs suggests that the particles were in the vasculature of the lungs.

\section{Discussion}

Our ICG-NCs spontaneously self-assemble via green chemistry, are fabricated with tunable diameters in the range of $\approx 60 \mathrm{~nm}$ to $1 \mu \mathrm{m}$, and the presence of amine groups on the surface of the constructs enables the covalent attachment of various moieties for molecular targeting.

To suppress the propensity of the ICG-NCs for nonspecific adsorption of proteins, we coated them with PEG, which is widely used to produce bio-inert surfaces by suppressing nonspecific interactions. ${ }^{46}$ To attain stability of the passivation coating, we covalently grafted the PEG chains to the amines on the surface of the nanoparticles. Employing chemical reactions that proceed through small intermediates with quantitative yields, such as reductive amination, ensures sufficiently well-packed PEG layers. ${ }^{47}$

The bio-inertness of the PEG coatings strongly depends on the length of the polymer chains. Our goal encompassed not only suppressing molecular-level nonspecific interactions - which can be achieved even with ethylene glycol oligomers (ie, with three to six repeating ethylene glycol units) - but also preventing nonspecific adhesion at mesoscopic (micron and sub-micron) levels. Coatings of PEG polymers that are too short would not provide sufficient separation between the nanoparticles and surfaces, to which they have tendency to be nonspecifically adsorbed by. Concurrently, PEG chains that are too long would result in layers in which the polymer termini have non-helical "mushroom" conformation that compromises the ability of such coatings to suppress nonspecific interactions. ${ }^{48}$ Using force measurements, as implemented with magnetic pullers, we previously determined that PEG with a $\mathrm{MW}$ of $5 \mathrm{kDa}-$ among the PEG molecules investigated with MW ranging between 1 and $20 \mathrm{kDa}$ - provided the optimal covalently grafted coatings for suppressing nonspecific adhesion. ${ }^{49}$ The higher flexibility and mobility of low MW PEG chains result in higher entropic repulsion between proteins and the surface.$^{50,51}$ Our in vitro studies confirmed the choice of PEG $5 \mathrm{kDa}$ for passivation coatings of ICG-NCs and for impeding their uptake by macrophages. ${ }^{10}$ Conversely, reduced flexibility of high MW PEG chains, due to the entrapment 
of long chains, induces low protein resistivity. In this study, we examined the biodistribution of ICG-NCs coated with a passivation coating of PEG $5 \mathrm{kDa}$.

In previous studies by other groups, ICG-containing constructs composed of poly(DL-lactic-co-glycolic-acid) (PLGA) whose surfaces were functionalized by both PEG and folic acid were administered into tumor-bearing mice. ${ }^{52,53}$ While these studies demonstrated the efficacy of targeted fluorescence imaging of tumors using functionalized ICGcontaining constructs, they did not isolate the effects of ICG encapsulation - and the additional PEGylation of the constructs in the absence of targeting moieties such as folic acid - in modulating the biodistribution of encapsulated ICG in a non-diseased mammalian system.

In another study by Zheng et al, ICG was entrapped within PLGA, and this construct was in turn coated with a lipid $2 \mathrm{kDa}$ PEG-folic acid assembly. ${ }^{53}$ Although the investigators reported the utility of these structures in targeted fluorescent imaging of tumor-bearing mice, the lipid $2 \mathrm{kDa}$ PEG-folic acid assembly was not covalently attached to the inner PLGA construct. Further, since PEG was simply a linker between the lipid and the folic acid, its role in suppressing nonspecific interactions is not clear.

Saxena et al investigated the biodistribution of PLGA nanoparticles entrapping ICG in healthy mice. ${ }^{40}$ Although these constructs were not PEGylated, the investigators reported that ICG encapsulation into these constructs resulted in ICG levels that were nearly six times higher within plasma at 1 hour post-tail vein injection in mice, as compared with those resulting from non-encapsulated ICG. In a previous study, our group reported on the biodistribution of ICG-NCs coated non-covalently with dextran or $10 \mathrm{~nm}$ ferromagnetic iron oxide nanoparticles, themselves individually coated with PEG, in healthy mice. ${ }^{35}$ The halflife of these particles in blood and their circulation kinetics appeared unaffected compared with free ICG, presumably due to the non-covalent attachment of the coating materials. Therefore, to the best of our knowledge, the study presented here is the first to have aimed at characterizing the biodistribution of encapsulated ICG in a construct system composed of an inner ICG core entrapped by a polymeric shell with covalent outer surface PEGylation by both in vivo quantitative fluorescence imaging and subsequent analysis of various extracted organs.

Consistent with Saxena et al's results, ${ }^{40}$ we observed a similar increase in ICG levels within plasma at 1 hour postinjection in the case of the PEGylated ICG-NCs (Figure 7C). We did not observe any significant differences in plasma ICG levels at 1 hour post-injection when ICG was administered in non-encapsulated form or as non-PEGylated ICG-NCs.

Higher ICG levels within various organs, including the spleen, heart, lungs, and kidneys, were reported by Saxena et al when ICG was delivered in the PLGA-entrapped form. These investigators also reported that, as early as 5 minutes post-injection, the amount of ICG within the liver was nearly 2.5 times greater when it was administered in the PLGA-entrapped form compared with when it was administered as free ICG. This result suggests that entrapment of ICG within the PLGA construct was not quite effective in delaying the accumulation of ICG within the liver. In comparison, in our study, we detected higher ICG levels within the liver at 30 minutes post-injection of both non-PEGylated and PEGylated ICG-NCs (Figure 7B).

In addition to the lack of PEGylation in the PLGA constructs, another factor that may be responsible for the differences between our results and those of Saxena et al is the size of the constructs. The mean diameter of the PLGA constructs was reported to be $300 \mathrm{~nm}$, nearly three times larger than the constructs used in our study. It has been indicated that PEGylated nanoparticles smaller than $100 \mathrm{~nm}$ have reduced plasma protein adsorption on their surfaces, and the blood clearance of such nanoparticles is slower than for larger $(>100 \mathrm{~nm})$ nanoparticles. ${ }^{31,54}$

Our results show that the covalent coating of ICG-NCs with $5 \mathrm{kDa}$ PEG can increase the blood circulation time of ICG. Our findings are consistent with reports by Ballou et $\mathrm{al}^{55}$ and Ohno et al, ${ }^{56}$ who have indicated prolonged systemic circulation of PEGylated silica nanoparticles in vivo. Ballou et al have reported a dramatic increase in the circulating half-life of core-shell zinc sulfide-cadmium selenide quantum dots coated with PEG (5000 Da). Their results have demonstrated that the extended circulation half-life of these particles is nearly 70 minutes compared with that of quantum dots coated with shorter length (750 Da and 3,400 Da) PEG chains, which had a half-life of less than 12 minutes. ${ }^{55}$ Ohno et al have reported prolonged blood halflife ( 20 hours) of PEGylated silica nanoparticles in healthy mice, with preferentially high accumulation in tumor tissue when injected into tumor-bearing mice. ${ }^{56}$ Shah et al studied the effect of PEG coating on the blood retention of gold nanoparticles. They indicated that the longer the particles stayed in the systemic circulation, the greater was the chance of accumulation in the tumor. ${ }^{57}$

The prolonged vascular circulation time of ICG as mediated by its encapsulation in these constructs may open up new possibilities for further clinical applications of ICG, which 
currently remain limited due to its rapid clearance from the vasculature and accumulation in the liver. The presence of ICG combined with the ability to functionalize the capsules provides the potential for ICG-NCs to serve as theranostic materials for the targeted optical imaging of specific molecular biomarkers of a disease and phototherapy. Previously, our group reported the targeted fluorescence imaging of head and neck squamous cells, cervical squamous cells, and breast cancer cells, with various expression levels of epidermal growth factor receptor (EGFR) using anti-EGFR-conjugated ICG-NCs. ${ }^{9}$ We have also reported the targeted fluorescent imaging of ovarian cancer cells using anti-human EGFR 2 functionalized ICG-NCs. ${ }^{58}$

ICG-NCs can potentially be used as a phototherapeutic agent by generating heat in response to laser irradiation. In a previous study, ${ }^{59}$ our group investigated the heat-generating capability of ICG-NCs in response to $808 \mathrm{~nm}$ laser irradiation and demonstrated the ability of ICG-NC suspensions to produce temperature rises to $\approx 80^{\circ} \mathrm{C}$. In another study, ${ }^{11}$ using a tissue phantom consisting of chicken breast to simulate normal tissue and an embedded gelatin cylinder (to simulate abnormal vasculature mass) loaded with ICG-NCs, we observed a nearly $20^{\circ} \mathrm{C}$ temperature rise within the gelatin cylinder at a depth of $3 \mathrm{~mm}$ below the surface in response to laser irradiation at $808 \mathrm{~nm}$ with an incident power of $4.2 \mathrm{~W}$. These results demonstrate the ability of ICG-NCs to induce a temperature rise in response to laser irradiation when embedded within an optically turbid tissue-like structure. In another study, ${ }^{9}$ we demonstrated the capability of ICG-NCs coated with antiEGFR to destroy cancer cell lines photothermally.

\section{Conclusion}

In this study, the biodistribution of ICG-NCs coated with $5 \mathrm{kDa}$ PEG in mice, as assessed by whole-body fluorescent imaging and individual organ uptake analysis, indicated that these particles have a prolonged vascular circulation time and delayed hepatic accumulation in comparison to uncoated constructs and non-encapsulated ICG. Thus, the encapsulation of ICG in such PEGylated constructs may potentially extend the current clinical applications of ICG because of its increased bioavailability.

\section{Acknowledgment}

This work was supported in part by grants from the National Science Foundation (CBET-1144237, CBET-0923408), Bourns College of Engineering, Bioengineering Center at the University of California, Riverside (UCR), and a student summer research grant from American Society for Laser Medicine and Surgery. The animal imaging was performed at the Institute for Integrative Genome Biology at UCR. We are grateful for the support provided by the Central Facility for Advanced Microscopy and Microanalysis at UCR. We thank Mr Andrew Reimer, a member of Bahman Anvari's group, for proofreading the manuscript.

\section{Disclosure}

The authors report no conflicts of interest in this work.

\section{References}

1. Frangioni J. In vivo near-infrared fluorescence imaging. Curr Opin Chem Biol. 2003;7(5):626-634.

2. Peer D, Karp JM, Hong S, Farokhzad OC, Margalit R, Langer R. Nanocarriers as an emerging platform for cancer therapy. Nat Nanotechnol. 2007;2(12):751-760.

3. Sevick-Muraca EM, Houston JP, Gurfinkel M. Fluorescence-enhanced, near infrared diagnostic imaging with contrast agents. Curr Opin Chem Biol. 2002;6(5):642-650.

4. Ntziachristos V, Bremer C, Weissleder R. Fluorescence imaging with near-infrared light: new technological advances that enable in vivo molecular imaging. Eur Radiol. 2003;13(1):195-208.

5. He X, Gao J, Gambhir SS, Cheng Z. Near-infrared fluorescent nanoprobes for cancer molecular imaging: status and challenges. Trends Mol Med. 2010;16(12):574-583.

6. Kim S, Lim YT, Soltesz EG, et al. Near-infrared fluorescent type II quantum dots for sentinel lymph node mapping. Nat Biotechnol. 2004;22(1):93-97.

7. Yaseen MA, Yu J, Wong MS, Anvari B. Stability assessment of indocyanine green within dextran-coated mesocapsules by absorbance spectroscopy. J Biomed Opt. 2007;12(6):064031.

8. Yaseen MA, Yu J, Wong MS, Anvari B. In-vivo fluorescence imaging of mammalian organs using charge-assembled mesocapsule constructs containing indocyanine green. Opt Express. 2008;16(25):20577-20587.

9. Yu J, Javier D, Yaseen MA, et al. Self-assembly synthesis, tumor cell targeting, and photothermal capabilities of antibody-coated indocyanine green nanocapsules. J Am Chem Soc. 2010;132(6):1929-1938.

10. Bahmani B, Gupta S, Upadhyayula S, Vullev VI, Anvari B. Effect of polyethylene glycol coatings on uptake of indocyanine green loaded nanocapsules by human spleen macrophages in vitro. J Biomed Opt. 2011;16(5):051303.

11. Yaseen MA, Yu J, Wong MS, Anvari B. Laser-induced heating of dextrancoated mesocapsules containing indocyanine green. Biotechnol Prog. 2007;23(6):1431-1440.

12. Yannuzzi LA. Indocyanine green angiography: a perspective on use in the clinical setting. Am J Ophthalmol. 2011;151(5):745-751. e1.

13. Ross A, Ross AH, Mohamed Q. Review and update of central serous chorioretinopathy. Curr Opin Ophthalmol. 2011;22(3):166-173.

14. Mantel I, Uffer S, Zografos L. Peripheral exudative hemorrhagic chorioretinopathy: a clinical, angiographic, and histologic study. Am J Ophthalmol. 2009;148(6):932-938. e1.

15. Tanaka E, Chen FY, Flaumenhaft R, Graham GJ, Laurence RG, Frangioni JV. Real-time assessment of cardiac perfusion, coronary angiography, and acute intravascular thrombi using dual-channel nearinfrared fluorescence imaging. J Thorac Cardiovasc Surg. 2009;138(1): 133-140.

16. El-Desoky A, Seifalian AM, Cope M, Delpy DT, Davidson BR. Experimental study of liver dysfunction evaluated by direct indocyanine green clearance using near infrared spectroscopy. British J Surg. 1999;86(8):1005-1011. 
17. Hirano A, Kamimura M, Ogura K, et al. A comparison of indocyanine green fluorescence imaging plus blue dye and blue dye alone for sentinel node navigation surgery in breast cancer patients. Ann Surg Oncol. 2012;19(13):4112-4116.

18. Kitai T, Inomoto T, Miwa M, Shikayama T. Fluorescence navigation with indocyanine green for detecting sentinel lymph nodes in breast cancer. Breast Cancer. 2005;12(3):211-215.

19. Miyashiro I, Miyoshi N, Hiratsuka M, et al. Detection of sentinel node in gastric cancer surgery by indocyanine green fluorescence imaging comparison with infrared imaging. Ann Surg Oncol. 2008;15(6): 1640-1643.

20. Nimura H, Narimiya N, Mitsumori N, Yamazaki Y, Yanaga K, Urashima M. Infrared ray electronic endoscopy combined with indocyanine green injection for detection of sentinel nodes of patients with gastric cancer. Br J Surg. 2004;91(5):575-579.

21. Uhara H, Yamazaki N, Takata M, et al. Applicability of radiocolloids, blue dyes and fluorescent indocyanine green to sentinel node biopsy in melanoma. J Dermatol. 2012;39(4):336-338.

22. Van der Vorst JR, Schaafsma BE, Verbeek FPR, et al. Randomized comparison of near-infrared fluorescence imaging using indocyanine green and $99(\mathrm{~m})$ technetium with or without patent blue for the sentinel lymph node procedure in breast cancer patients. Ann Surg Oncol. 2012; 19(13):4104-4111.

23. Moretó M. Diagnosis of esophagogastric tumors. Endoscopy. 2003; 35(1):36-42.

24. Yamamoto T, Narushima M, Doi K, et al. Characteristic indocyanine green lymphography findings in lower extremity lymphedema: the generation of a novel lymphedema severity staging system using dermal backflow patterns. Plast Reconstr Surg. 2011;127(5):1979-1986.

25. Yamamoto T, Yamamoto N, Doi K, et al. Indocyanine green-enhanced lymphography for upper extremity lymphedema: a novel severity staging system using dermal backflow patterns. Plast Reconstr Surg. 2011;128(4):941-947.

26. Yoneya S, Saito T, Komatsu Y, Koyama I, Takahashi K, Duvoll-Young J. Binding properties of indocyanine green in human blood. Invest Ophthalmol Vis Sci. 1998;39(7):1286-1290.

27. Altinoğlu EI, Adair JH. Near infrared imaging with nanoparticles Wiley Interdiscip Rev Nanomed Nanobiotechnol. 2010;2(5):461-477.

28. Desmettre TJ, Soulie-Begu S, Devoisselle JM, Mordon SR. Diode laserinduced thermal damage evaluation on the retina with a liposome dye system. Lasers Surg Med. 1999;24(1):61-68.

29. Rodriguez VB, Henry SM, Hoffman AS, Stayton PS, Li X, Pun SH. Encapsulation and stabilization of indocyanine green within poly(styrene-alt-maleic anhydride) block-poly(styrene) micelles for near-infrared imaging. J Biomed Opt. 2013;13(1):014025.

30. Sharma P, Bengtsson NE, Walter GA, et al. Gadolinium-doped silica nanoparticles encapsulating indocyanine green for near infrared and magnetic resonance imaging. Small. 2012;8(18):2856-2868.

31. Alexis F, Pridgen E, Molnar LK, Farokhzad OC. Factors affecting the clearance and biodistribution of polymeric nanoparticles. Mol Pharm. 2008;5(4):505-515.

32. Cao Z, Jiang S. Super-hydrophobic switterionic poly(carboxybetaine) and amphiphilic non-ionic poly(ethylene glycol) for stealth nanoparticles. Nano Today. 2012;7(5):404-413.

33. Bahmani B, Gupta S, Vullev V, Anvari B. Uptake of PEGylated indocyanine green loaded nanocapsules by cells of reticuloendothelial system. In: Achilefu S, Raghavachari R, editors. Reporters, Markers, Dyes, Nanoparticles, and Molecular Probes for Biomedical Applications III. Proceedings of SPIE Volume 7910. Bellingham, WA: SPIE; 2011:79101C.

34. Bahmani B, Jung B, Gupta S, Anvari B. Cellular uptake of polymeric nanocapsules loaded with ICG by human blood monocytes and human spleen macrophages. In: Achilefu S, Raghavachari R, editors. Reporters, Markers, Dyes, Nanoparticles, and Molecular Probes for Biomedical Applications III. Proceedings of SPIE Volume 7576. Bellingham, WA: SPIE; 2010:75761Q.
35. Yaseen MA, Yu J, Jung B, Wong MS, Anvari B. Biodistribution of encapsulated indocyanine green in healthy mice. Mol Pharm. 2009;6(5): 1321-1332.

36. Ebert B, Licha K. Cyanine dyes as contrast agents for near-infrared imaging in vivo: acute tolerance, pharmacokinetics, and fluorescence imaging. J Biomed Opt. 2011;16(6):066003.

37. Rasband WS. ImageJ [software]. Bethesda, MD: US National Institutes of Health; 1997-2012.

38. Houston JP, Ke S, Wang W, Li C, Sevick-Muraca EM. Quality analysis of in vivo near-infrared fluorescence and conventional gamma images acquired using a dual-labeled tumor-targeting probe. J Biomed Opt. 2005;10(5):054010.

39. Sakka SG, Koeck H, Meier-Hellmann A. Measurement of indocyanine green plasma disappearance rate by two different dosages. Intensive Care Med. 2004;30(3):506-509.

40. Saxena V, Sadoqi M, Shao J. Polymeric nanoparticulate delivery system for Indocyanine green: biodistribution in healthy mice. Int J Pharm. 2006;308(1-2):200-204.

41. Desmettre T, Devoisselle JM, Mordon S. Fluorescence properties and metabolic features of indocyanine green (ICG) as related to angiography. Surv Ophthalmol. 2000;45(1):15-27.

42. Cui Y, König J, Leier I, Buchholz U, Keppler D. Hepatic uptake of bilirubin and its conjugates by the human organic anion transporter SLC21A6. J Biol Chem. 2001;276(13):9626-9630.

43. Cesta MF. Normal structure, function, and histology of the spleen. Toxicol Pathol. 2006;34(5):455-465.

44. Mebius RE, Kraal G. Structure and function of the spleen. Nat Rev Immunol. 2005;5(8):606-616.

45. Card JW, Zeldin DC, Bonner JC, Nestmann ER. Pulmonary applications and toxicity of engineered nanoparticles. Am J Physiol Lung Cell Mol Physiol. 2008;295(3):L400-L411.

46. Leckband D, Sheth S, Halperin A. Grafted poly(ethylene oxide) brushes as nonfouling surface coatings. J Biomater Sci Polymer Edn. 1999;10(10):1125-1147.

47. Wan J, Thomas MS, Guthrie S, Vullev VI. Surface-bound proteins with preserved functionality. Ann Biomed Eng. 2009;37(6): 1190-1205.

48. Gref R, Lück M, Quellec P, et al. "Stealth" corona-core nanoparticles surface modified by polyethylene glycol (PEG): influences of the corona (PEG chain length and surface density) and of the core composition on phagocytic uptake and plasma protein adsorption. Colloids Surf B Biointerfaces. 2000;18(3-4):301-313.

49. Upadhyayula S, Quinata T, Bishop S, et al. Coatings of polyethylene glycol for suppressing adhesion between solid microspheres and flat surfaces. Langmuir. 2012;28(11):5059-5069.

50. Harder P, Grunze M, Dahint R, Whitesides GM, Laibinis PE. Molecular conformation in oligo (ethylene glycol) - terminated self-assembled monolayers on gold and silver surfaces determines their ability to resist protein adsorption. J Phys Chem B. 1998;102(2):426-436.

51. Xu Z, Holland NB, Marchant RE. Conformations of short-chain poly(ethylene oxide) lipopolymers at the air-water interface: a combined film balance and surface tension study. Langmuir. 2001;17(2): 377-383.

52. Ma Y, Sadoqi M, Shao J. Biodistribution of indocyanine green-loaded nanoparticles with surface modifications of PEG and folic acid. Int $J$ Pharm. 2012;436(1-2):25-31.

53. Zheng $\mathrm{C}$, Zheng $\mathrm{M}$, Gong $\mathrm{P}$, et al. Indocyanine green-loaded biodegradable tumor targeting nanoprobes for in vitro and in vivo imaging. Biomaterials. 2012;33(22):5603-5609.

54. Fang $\mathrm{C}$, Shi B, Pei YY, Hong MH, Wu J, Chen HZ. In vivo tumor targeting of tumor necrosis factor-alpha-loaded stealth nanoparticles: effect of MePEG molecular weight and particle size. Eur J Pharm Sci. 2006;27(1):27-36.

55. Ballou B, Lagerholm BC, Ernst LA, Bruchez MP, Waggoner AS. Noninvasive imaging of quantum dots in mice. Bioconjugate Chem. 2004;15(1):79-86. 
56. Ohno K, Akashi T, Tsujii Y, Yamamoto M, Tabata Y. Blood clearance and biodistribution of polymer brush-afforded silica particles prepared by surface-initiated living radical polymerization. Biomacromolecules. 2012;13(3):927-936.

57. Shah NB, Vercellotti GM, White JG, Fegan A, Wagner CR, Bischof JC. Blood-nanoparticle interactions and in vivo biodistribution: impact of surface PEG and ligand properties. Mol Pharm. 2012. Epub July 23.
58. Bahmani B, Vullev V, Anvari B. Development of anti-HER2 conjugated ICG-loaded polymeric nanoparticles for targeted optical imaging of ovarian cancer. In: Achilefu S, Raghavachari R, editors. Reporters, Markers, Dyes, Nanoparticles, and Molecular Probes for Biomedical Applications III. Proceedings of SPIE Volume 82. Bellingham, WA: SPIE; 2012:82330L.

59. Yu J, Yaseen MA, Anvari B, Wong MS. Synthesis of near-infrared-absorbing nanoparticle-assembled capsules. Chem Mater. 2007;(8):1476-1483.

\section{Publish your work in this journal}

The International Journal of Nanomedicine is an international, peerreviewed journal focusing on the application of nanotechnology in diagnostics, therapeutics, and drug delivery systems throughout the biomedical field. This journal is indexed on PubMed Central, MedLine, CAS, SciSearch $\AA$, Current Contents ${ }^{\circledR} /$ Clinical Medicine,
Journal Citation Reports/Science Edition, EMBase, Scopus and the Elsevier Bibliographic databases. The manuscript management system is completely online and includes a very quick and fair peer-review system, which is all easy to use. Visit http://www.dovepress.com/ testimonials.php to read real quotes from published authors. 